\title{
Alcohol Induced Rhabdomyolysis
}

\author{
Ali Reza Zahiroddin, ${ }^{1}$ and Zahra Rezaei ${ }^{1, *}$ \\ ${ }^{1}$ Behavioral Sciences Research Center of Psychiatry Department of Shahid Beheshti University of Medical Sciences, Imam Hossein Hospital, Tehran, IR Iran \\ "Corresponding author: Zahra Rezaei, Behavioral Sciences Research Center of Psychiatry Department of Shahid Beheshty Medical University, Imam Hossein Hospital, Tehran, IR \\ Iran. Tel: +98-2177551023, +98-9125407836, Fax: +98-2177551023, E-mail: z.rezaei1363@yahoo.com
}

Received 2015 December 11; Revised 2016 August 6; Accepted 2016 October 29

\begin{abstract}
Rhabdomyolysis is a common condition with potentially devastating complications, and only few therapeutic options are available for the treatment of acute Rhabdomyolysis. In this case, a 56-year-old male who had been drinking alcohol, presented to the emergency department for evaluation of slurred speech, ataxic gait, mood instability, and cognitive disorder. On admission, his creatine kinase (CK) level was $2882 \mathrm{U} / \mathrm{L}$. Despite aggressive fluid repletion, his CK level continued to increase, peaking at 11350 U/L. On administration and after the fluid transfusion with isotonic saline, his CK levels and clinical symptoms improved dramatically. We report this case as it is a rare condition of rhabdomyolysis due to alcohol, with the intent to highlight its clinical features and paraclinical finding.
\end{abstract}

Keywords: Alcohol, Creatine Kinase, Rhabdomyolysis

\section{Introduction}

Rhabdomyolysis is defined as skeletal muscle injury that leads to the lysis of muscle cells and the leakage of myocyte contents into the extracellular compartments (1). The presenting clinical features are myalgia, myoglobinuria, and elevated serum creatine kinase (1-3). The presence of myoglobin in the urine will produce a dark redbrown pigmentation (3-5). The function of CK is to convert myocyte creatine phosphate into high-energy phosphate groups (adenosine triphosphate) used in energy requiring reactions (6). Degradation of approximately 200 grams of muscle can cause an increase in serum CK $(3,6)$. When the massive myocyte breakdown of cell membranes occurs, other intracellular constituents are released besides myoglobin and CK (1-3). Approximately 150 grams of muscle necrosis will release more than $15 \mathrm{mmol}$ of potassium (13). The resulting hyperkalemia may increase the risk for cardiac arrhythmia and complicate an existing acute renal failure (1-3). Rhabdomyolysis is caused by a wide variety of conditions, including trauma, drugs, viruses, and metabolic disorders $(7,8)$. Rhabdomyolysis is a potentially fatal condition, with a mortality rate of approximately $8 \%$ (7). The most common complication of rhabdomyolysis is renal failure, accounting for 7 to $10 \%$ of all cases of acute kidney injury $(1,7)$.

Aggressive fluid 8 repletion is the first-line treatment for rhabdomyolysis, which reduces the accumulation of toxic intracellular contents caused by the rapid break- down of muscle and subsequent renal damage $(7,9)$. Rapid fluid transfusion with isotonic saline should be initiated as early as possible to preserve renal function while monitoring urine output and level of $\operatorname{K}$ and $\operatorname{CK}(7,9)$. The infusion can be adjusted to maintain urine output at 300 $\mathrm{mL} / \mathrm{h}$ (7). Furthermore, fluid management, and medications that may be prescribed include diuretics and bicarbonate, but some patients may need kidney dialysis $(7,9)$.

\section{Case Presentation}

A 56 year-old- male, with no history of hospitalization presented to the emergency department of Imam-Hossein hospital.

The chief complaint of this patient was ataxic gait, and the main reason for referral was fluctuation of consciousness. Abnormal symptoms were as follows: Slurred speech, nystagmus, mood instability, and |cognitive disorder, including fluctuation of consciousness and reduction of environmental awareness. Due to financial debts and difficulties and its relevant anxiety and sleep disturbance, the patient had consumed alcoholic beverages with approximately $70 \%$ alcohol by volume (ABV) as of five days prior to admission.

Association symptoms findings included nausea, vomiting and visual darkening after alcohol consumption. He started drinking alcoholic beverages with $2 \% \mathrm{ABV}$ and increased them to $70 \% \mathrm{ABV}$ in the last 30 years; he especially 
drank more palpably in the last 10 recent years, but he had not experienced any episodes with similar symptoms.

Determining the cause of fluctuating level of consciousness and evaluating his psychiatric history at the beginning stages were impossible. Nevertheless, slurred speech, mood instability, fluctuating level of consciousness, reduction of environmental awareness, nausea, vomiting and visual darkening and nystagmus were mentioned in the patient after he drank alcohol.

According to his family history and because of his financial problems and debts, other symptoms were also detected, which were as follows: Irritable mood, and preoccupation about debt, sleep disturbance, loss of interest, loss of energy, diminished ability to think from the last month and consumption of alcoholic beverages with approximately $70 \% \mathrm{ABV}$ daily as of five days prior to his admission.

In his past psychiatric history, he had numerous periods of irritable mood, inflated self-esteem, decreased need for sleep (only 3 - 4 hours), increased goal directed activities, and worked with excessive involvement in activities and painful consequence.

These symptoms continued for at least four days as of 30 years ago. He had numerous periods of depressive symptoms, which were as follows: Loss of interest and loss of energy and depressed mood, with diminished ability to think. However, no thoughts of death and suicidal ideation were detected. The results of mental status examinations were as follows: A middle-aged man whose appearance and real age was proportionate, his visual and verbal contact was partially with semi- cooperative attitude, psychomotor restlessness, slurred speech, irritable mood and appropriate affect and normal thought. However, he had no death wish, suicidal idea, delusion or hallucination.

The MMSE test revealed the following results: Poor registration (Score 1), poor attention and calculation (serial seven, Score 1), impaired time orientation (Score 1), but good orientation to place and partially to person (Score 3), impaired recall (Score 0), good score in language (Score 2).

The patient did not cooperate for other examinations of MMSE.

The pupil was of normal size, and double reactive.

He had no medical history (seizure, head trauma, hypertension), or drug history, except for Chlordiazepoxide tablet, $5 \mathrm{mg}$ daily.

With respect to family history, his mother had major depression disorder.

The patient started drinking alcoholic beverages with $2 \% \mathrm{ABV}$ as of 30 years ago, and his consumption was infrequent. Nevertheless, he needed excessive dosage from the past 10 years because of gambling and debt, and mood disturbance, approximately $40 \%-50 \% \mathrm{ABV}$, but he did not ex- perience episodes of similar symptoms. His childhood and adulthood history was not clear. He married 30 years ago, and his relationship with his wife has often been stormy with frequent fighting from the early years of marriage; he has two sons. He was a real estate agent, without job change, and without past criminal or judicial involvement.

After admission in the emergency department, his vital signs were as follows: Temperature: 37, heart rate: 96/minutes, respiratory rate: 18/minutes, blood pressure: 130/80 mmHg. Laboratory studies on admission revealed the following results: Complete blood cell count was normal. Complete metabolic profile yielded the following results: Sodium:139 mmol/L; potassium: $3 / 3 \mathrm{mmol} / \mathrm{L}$; blood urea nitrogen:14 mg/dL; creatinine: $1 / 3 \mathrm{mg} / \mathrm{dL}$; glucose: 120 $\mathrm{mg} / \mathrm{dL}$; calcium: $8 / 5 \mathrm{mg} / \mathrm{dL}$; and aspartate amino transferase: 53U/L. Creatine kinase (2882U/L) and urinalysis reported darkening of the urine (brown); SG: greater than 1/027; protein value: $2+$; blood value: $3+$; urobilinogen level: 2+ without glucose, nitrites; RBC: 12 - 18/hpf; WBC: 4 - 6/hpf; and Ep-cell: 4-6/hpf. Brain CT-Scan was done, and its results were normal.

He had received $2 \mathrm{~L}$ of normal saline in the emergency department, followed by a continuous infusion of 200 $\mathrm{mL} / \mathrm{h}$ of normal saline while in the hospital. The patient's symptoms worsened despite fluid repletion. On the second day, he was unable to ambulate or sit up in the bed secondary to muscle pain and weakness. After 24 hours of admission, with normal consciousness level, he was moved to the psychiatric department. The patient was able to ambulate, but muscle pain and weakness existed. The laboratory tests were done daily on the following days.

CK level after one day elevated to $11350 \mathrm{U} / \mathrm{L}$, and urine darkened to coffee color despite fluid repletion. Creatinine and blood urea nitrogen remained within normal limits. Potassium raised to $5 / 8 \mathrm{mmol} / \mathrm{L}$, and key oxalate powder and sorbitol powder were prescribed. Aspartate Amino Transferase was $262 \mathrm{U} / \mathrm{L}$, and Alanine Amino Transferase was 102U/L. Braine MRI without contrast was done, but no pathology was found. The lab test results demonstrated a decrease of the CK level in the third day; CK level decreased to $6820 \mathrm{U} / \mathrm{L}$ after four days and it decreased to $3375 \mathrm{U} / \mathrm{L}$ after five days. Finally, the CK level decreased to $389 \mathrm{U} / \mathrm{L}$ after eight days.

He was discharged with the diagnosis of cyclothymia, numerous periods of hypomania (irritable mood, inflated self-esteem, decreased need for sleep (only 3 - 4 hours), and an increase in goal directed activity at work. These symptoms lasted for at least four days during the past 30 years, and with numerous periods of depressive symptoms such as loss of interest and loss of energy and depressed mood with diminished ability to think (with DSM4-IV-TR Criteria). 
Other diagnosis included alcohol intoxication (DSM4IV-TR Criteria), which caused slurred speech, mood instability, consciousness fluctuation, reduction of environmental awareness, ataxic gait, nausea, vomiting, and nystagmus after the consumption of alcoholic beverages with $70 \% \mathrm{ABV}$.

\section{Discussion}

This case was challenging as the patient responded slowly to the first-line therapy. No standardized secondline treatment was available for rhabdomyolysis, and he was at a substantial risk of acute renal injury (1-3). The risk of renal failure in these cases is directly proportional to CK levels (3). His CK level increased to more than $11000 \mathrm{U} / \mathrm{L}$, an abnormally high value, even in acute cases of alcohol intoxication. Thus, our patient was at high risk of end-organ damage with limited treatment options.

Ethanol causes rhabdomyolysis through disruption of Adenosine triphosphates pump function, break down of the muscle membrane, alteration of the sarcoplasmic reticulum, sodium permeability and disrupting calcium homeostasis, inducing cytokine releasing the levels of "interleukin (IL) 1, IL-2, IL-6", and" tumor necrosis factor $\alpha$ ” (812).

The muscle damage was caused by activated neutrophils that infiltrate the damaged muscle tissue and release protease in response to liberated toxic cellular contents $(9,12)$. Considering the induction of immune pathway by ethanol, one hypothesis should be contemplated: Corticosteroid administration helped diminish the inflammatory exacerbation of muscle damage and potentially decreased secondary immune-mediated muscle damage.

A high dose of corticosteroids is likely justified in acute cases of rhabdomyolysis given the additional benefit of short-term T-cell suppression compared with low-dose administration (11).

Data on the effectiveness of corticosteroids in treating rhabdomyolysis are limited (11). However, the time course of corticosteroid administration and the dramatic decreases in CK level and symptom improvement suggest a pharmacologic effect.

In fact, to our knowledge, only three cases of methylprednisolone administration to treat rhabdomyolysis have been reported, and the patients' CK level decreased abruptly after administering methylprednisolone. Methylprednisolone is a good choice to treat inflammatory diseases, and inflammatory processes could be the possible reason for the beneficial treatment of rhabdomyolysis due to alcohol (11).
In our treatment, the patient received $2 \mathrm{~L}$ of normal saline in the emergency department, followed by a continuous infusion of $200 \mathrm{~mL} / \mathrm{h}$ of normal saline, and key oxalate powder and sorbitol powder were prescribed. However, we did not prescribe corticosteroid because of the decrease in the CK level and the risk for mood disturbance.

After the causes of this condition were induced with alcohol, because of the muscle compression and muscular ischemia and the rapid release of muscle cell contents into circulation, the conditions of these patients might become more complicated with hyperkalemia and acute renal failure and multi organ failure $(3,7)$.

This complication is diagnosed by the elevation of the CK level with NL CK.MB $(3,7,13)$. After the diagnosis of rhabdomyolysis, a rapid fluid transfusion should be initiated as early as possible to preserve renal function while monitoring urine output and level of potassium and total CK. (11).

All patients who are diagnosed with rhabdomyolysis should be hospitalized for rapid replacement therapy, with isotonic saline as the treatment of choice even if there is no evidence of dehydration $(3,7)$.

The infusion rate can be adjusted to maintain urine output at $300 \mathrm{~mL} /$ hour (7). However, fluid management does not address the underlying causes of myotoxicity or minimize muscle damage (11). Some have suggested the use of mannitol or bicarbonate in conjunction with fluid repletion in the treatment of rhabdomyolysis $(3,7,11)$. Studies have shown a mild benefit with urine alkalization in reducing kidney injury, particularly when the alkalinizing agent is administered early in the course of rhabdomyoly$\operatorname{sis}(1,3,11)$.

The differential diagnosis should be considered such as neuroleptic malignant syndrome that is characterized by fever, muscle rigidity, altered mental status, and autonomic dysfunction; the first two signs are the hallmarks of this diagnosis, which were not detected in our patient. In addition, there were no signs of catatonic state, including stupor, posturing, waxy flexibility, stereotypy, mutism, echolalia, echopraxia, so this diagnosis was ruled out (12).

\subsection{Conclusions}

Rhabdomyolysis is a common condition that, if left untreated, can have devastating complications, including renal failure and death. This complication is caused by a wide variety of conditions, including trauma, drugs, viruses, and metabolic disorders. Few treatment options are available for unresponsive patients to first line fluid management. Rare cases have been reported in conditions like alcohol-induced rhabdomyolysis. Due to the induction of immune pathway, corticosteroids may break the cycle of the primary muscle toxicity, followed by inflammation -induced muscle damage, and it may be a viable treatment op- 
tion in this case. We believe that increased awareness and a high level of apprehension will assist physicians in diagnosing and treating non- traumatic rhabdomyolysis in patients who present with malaise, weakness, and history of alcohol abuse.

\subsection{Consent}

During the evaluation report, we obtained informed consent from the patient for publication and for a follow up process if necessary.

\section{Acknowledgments}

We are highly grateful to the patient and the behavioral sciences research center of psychiatry department of Shahid Beheshti University of Medical Sciences and Imam Hossein hospital.

\section{Footnotes}

Authors' Contribution: Alireza Zahiroddin conceived and designed the evaluation. Zahra Rezaei collected the clinical data. Alireza Zahiroddin and Zahra Rezaei interpreted the clinical data. Zahra Rezaei drafted the manuscript. Alireza Zahiroddin and Zahra Rezaei revised it critically for important intellectual content. Both authors read and approved the final manuscript.

Declaration of Interest: None declared.

Funding/Support: None declared.

\section{References}

1. Bosch X, Poch E, Grau JM. Rhabdomyolysis and acute kidney injury. N Engl J Med. 2009;361(1):62-72. doi: 10.1056/NEJMra0801327. [PubMed: 19571284].
2. Vanholder R, Sever MS, Erek E, Lameire N. Rhabdomyolysis. J Am Soc Nephrol. 2000;11(8):1553-61. [PubMed: 10906171].

3. Bhavsar P, Rathod KJ, Rathod D, Chamania CS. Utility of Serum Creatinine, Creatine Kinase and Urinary Myoglobin in Detecting Acute Renal Failure due to Rhabdomyolysis in Trauma and Electrical Burns Patients. Indian J Surg. 2013;75(1):17-21. doi: 10.1007/s12262-012-0451-6. [PubMed: 24426377].

4. Sulowicz W, Walatek B, Sydor A, Ochmanski W, Milkowski A, Szymczakiewicz-Multanowska A, et al. Acute renal failure in patients with rhabdomyolysis. Med Sci Monit. 2002;8(1):CR24-7. [PubMed: 11782676].

5. Lima RS, da Silva Junior GB, Liborio AB, Daher Ede F. Acute kidney injury due to rhabdomyolysis. Saudi J Kidney Dis Transpl. 2008;19(5):7219. [PubMed: 18711286].

6. Hall JE. Guyton and Hall textbook of medical physiology. Elsevier Health Sciences; 2015.

7. Cervellin G, Comelli I, Lippi G. Rhabdomyolysis: historical background, clinical, diagnostic and therapeutic features. Clin Chem Lab Med. 2010;48(6):749-56. doi: 10.1515/CCLM.2010.151. [PubMed: 20298139].

8. Jankovic SR, Stosic JJ, Vucinic S, Vukcevic NP, Ercegovic GV Causes of rhabdomyolysis in acute poisonings. Vojnosanit Pregl. 2013;70(11):1039-45. [PubMed: 24397200].

9. Haas CE, Magram Y, Mishra A. Rhabdomyolysis and acute renal failure following an ethanol and diphenhydramine overdose. Ann Pharmacother. 2003;37(4):538-42. [PubMed: 12659612].

10. Su MS, Jiang Y, Yan XY, Zhao QH, Liu ZW, Zhang WZ, et al. Alcohol abuse-related severe acute pancreatitis with rhabdomyolysis complications. Exp Ther Med. 2013;5(1):189-92. doi: 10.3892/etm.2012.735. [PubMed: 23251265].

11. Antoon JW, Chakraborti C. Corticosteroids in the treatment of alcohol-induced rhabdomyolysis. Mayo Clin Proc. 2011;86(10):1005-7. doi: 10.4065/mcp.2011.0102. [PubMed: 21964178].

12. Voros V, Osvath P, Fekete S, Tenyi T. Elevated serum creatine kinase levels in psychiatric practice: differential diagnosis and clinical significance: A brief, practical guideline for clinicians. Int J Psychiatr Clin Pract. 2008;12(2):147-50.

13. Premru V, Kovac J, Ponikvar R. Use of myoglobin as a marker and predictor in myoglobinuric acute kidney injury. Ther Apher Dial. 2013;17(4):391-5. doi: 10.1111/1744-9987.12084. [PubMed: 23931877]. 